\title{
Decoherence of one-dimensional flying qubits due to their cross-talk and imperfections
}

\author{
D. V. Khveshchenko \\ Department of Physics and Astronomy, University of North Carolina, Chapel Hill, NC 27599
}

\begin{abstract}
We study decoherence of propagating spin-1/2 excitations in generic (non-integrable and/or disordered) spin chains. We find the relevant decoherence times to be shorter in both the nearcritical and diffusive regimes (if any), which fact might have important implications for the recently proposed spin chain-based implementations of quantum information processing.
\end{abstract}

In spite of its success in describing thermodynamics and, more recently, spin/thermal transport, the theory of quantum one-dimensional $(1 D)$ spin systems has been struggling to develop a systematic approach to such nonequilibrium problems as, e.g., the time evolution of a certain (in general, non-stationary) input state even in the simplest exactly solvable cases (see, e.g., Refs. [1]). However, it is exactly this kind of problems that have been brought about by the recent advents of spintronics and quantum information processing (QIP).

For example, many theoretical discussions of an abstract multi-qubit $(N \gg 1)$ register invoke a generic spin1/2 Hamiltonian [2]

$$
H=\sum_{a=x, y, z}\left(\sum_{i=1}^{N} B_{i}^{a} S_{i}^{a}+\sum_{i, j=1}^{N} J_{i j}^{a} S_{i}^{a} S_{j}^{a}\right)
$$

while the usually proposed architectures have the topology of a $1 D, 2 D$ or $3 D$ array with the nearest-neighbor (NN) inter-qubit couplings (despite a desireable reduction in the required number of qubits, the higher connectivity networks may not be that easy to assemble in practice).

In the QIP context, the issue of paramount importance is that of quantum coherence which, if no precautions are taken, can be easily destroyed by any coupling to an external dissipative environment. Moreover, even though in the absence of such a coupling the density matrix $\hat{\rho}(t)$ of the entire $N$-qubit system undergoes unitary evolution, any reduced density matrix (RDM) obtained by tracing out some of the degrees of freedom can still exhibit an ostensible decay caused solely by the qubits' "cross-talk".

In the QIP-related applications, one is primarily interested in the short-time behavior which strongly diminishes any chances of the Poincare recurrences of the initial quantum state that might occur in the noiseless limit at longer times. Furthermore, an arbitrarily weak coupling to a noisy environment eliminates any possible quantum states' revivals and makes this "deterministic decoherence" a truly irreversible behavior.

Contrary to the non-interacting case, however, the analysis of decoherence in a system of many coupled qubits described by Eq.(1) can no longer be carried out in terms of the conventional transverse/longitudinal relaxation times $\left(T_{1,2}\right)$ of individual on-site spins. Instead, an adequate discussion must start out by identifying the proper basis of delocalized spin- $1 / 2$ states with definite momenta (hereafter referred to as "spinons") that span the Hilbert space of the interacting multi-qubit system (1) and then proceed towards computing various $n$ spinon $(n=1,2, \ldots)$ RDMs given by the partial traces $\operatorname{Tr}_{1, \ldots, N-n} \hat{\rho}(t)$.

Besides providing an appropriate framework for the discussion of decoherence, the spinon basis can also offer a natural implementation of the notion of "flying qubits" proposed as a vehicle for performing such important QIP tasks as teleportation or quantum key distribution [2].

To this end, in the present paper we evaluate the spinon decoherence rates in several situations that might be of interest for QIP and ascertain the prospects of using spinons in the QIP protocols relying on the availability of flying qubits.

At first sight, the presence of unbound spinons in the spectrum of the Hamiltonian (1) may seem like a commonplace, as suggested by the possibility to readily cast Eq.(1) in the form of the lattice Hamiltonian of spin$1 / 2$ fermions with some short-ranged quartic interactions by applying a fermion representation of the spin operators $\left(\vec{S}_{i}=\frac{1}{2} \psi_{i \sigma}^{\dagger} \vec{\sigma}_{\sigma \sigma^{\prime}} \psi_{i \sigma^{\prime}}\right)$ complemented by a local single-occupancy constraint $\left(\psi_{i \sigma}^{\dagger} \psi_{i \sigma}=1\right)$. However, the answer to the question as to whether or not the "constituent" fermion operators $\psi_{i \sigma}$ correspond to any (possibly, approximate) eigenstates of the Hamiltonian (1) would strongly depend on the actual form of the latter.

The existence of deconfined spinons in higher dimensions (or a lack thereof) had been the subject of a long ongoing debate which was rekindled by the discovery of cuprates representing a new class of strongly correlated Mott insulators. Despite an extensive work on the topic, however, only a handful of solid arguments in favor of the occurrence of such excitations in a number of rather exotic models have been made so far (see, e.g., [3] and references therein).

In contrast, many 1D spin systems do conform to the picture of a "spinon Fermi liquid" (SFL) whose properties appear to be quite different from those of the spinordered states described in terms of the conventional spin-1 magnons. Incidentally, the interest in spin-chains as a promising layout of the practical quantum register has been bolstered by the reports [4] of a massive buildup of quantum entanglement at those points in the parameter space of Eq.(1) where the system undergoes quantum 
phase transitions.

It should be noted, however, that, depending on the qubits' physical makeup, the corresponding Eq.(1) may lack any particular spin symmetry, thus hampering the applicability of such customary 1D techniques as the Bethe ansatz, bosonization, and conformal field theory.

As an alternate approach that has been previously employed in various strongly correlated fermion systems, we adopt the semi-phenomenological spin-fermion model described by the action [5]

$$
\begin{gathered}
A=\sum_{k} \int \frac{d E}{(2 \pi)} \psi_{k \sigma}^{\dagger}\left(E-\epsilon_{k}\right) \psi_{k \sigma}+\sum_{q} \int \frac{d \omega}{(2 \pi)} S_{q}^{a} \chi_{a b}^{-1}(\omega, q) \\
S_{-q}^{b}+g \sum_{k, q} \int \frac{d E d \omega}{(2 \pi)^{2}} \psi_{k \sigma}^{\dagger} \hat{\sigma}_{\sigma \sigma^{\prime}}^{a} S_{q}^{a} \psi_{k+q, \sigma^{\prime}}
\end{gathered}
$$

written in terms of the continuum fermionic variables which are no longer subject to the local single-occupancy constraint, while their interactions are mediated by spin density fluctuations governed by the dynamical spin susceptibility $\chi^{a b}(\omega, q)=\sum_{i j} \int d t e^{i \omega t-i q|i-j|}<$ $S_{i}^{a}(t) S_{j}^{b}(0)>$.

The gapful fermion dispersion $\epsilon_{k}=\sqrt{v^{2} k^{2}+\Delta^{2}}$ accounts for the main effect of the processes of backward scattering, and the gap $\Delta \ll J$ is assumed to vanish at certain values of the microscopic parameters in Eq.(1).

Thus, the model (2) describes a gas of (for any $\Delta \neq 0$, massive) spinons that are still subject to a forward scattering, the singular part of which is controlled by the effective coupling constant $g$ measuring a deviation from the parent SFL state. In turn, the latter is thought to be composed of quasiparticles that can be adiabatically related to the one-spinon states $\left|k \sigma>=\psi_{k \sigma}^{\dagger}\right| 0>$ even in the presence of non-singular residual interactions that were omitted in Eq.(2).

Right at the quantum critical point (QCP) $\Delta=0$, and at all energies below a high-energy cutoff set by the average exchange coupling $J$ the $T=0$ susceptibility has a generic power-law form

$$
\chi_{Q C P}^{a b}(\omega, q)=\frac{\eta^{a b}(q) J^{2 \alpha_{a b}}}{\left(v^{2} q^{2}-\omega^{2}\right)^{\alpha_{a b}}}
$$

In the absence of spin-rotational invariance, the exponents $\alpha_{a b}$ and the prefactors $\eta^{a b}(q)$ depend on the direction in spin space. In the isotropic $(S U(2)-)$ or axially $(U(1)-)$ symmetric case, however, all (or some) of the numerators vanish at zero transferred momentum $q$ in compliance with the conservation of the corresponding component of the total spin.

At temperatures above the gap $(\Delta<T \lesssim J)$ the susceptibility demonstrates a characteristic "quantumcritical" (QC) behavior

$$
\chi_{Q C}^{a b}(\omega, q)=\frac{\chi_{0}^{a b}(T)}{q^{2} \xi^{2}-\left(\omega-i \gamma_{Q C}\right)^{2} / \Omega^{2}}
$$

where $v / \xi \sim \Omega \sim \gamma_{Q C} \propto T$ and $\chi_{0}^{a b}(T) \propto(J / T)^{2 \alpha_{a b}}[6]$.

Notably, the temperature-dependent relaxation rate $\gamma_{Q C}=\left.\frac{1}{2} \operatorname{Im} \frac{d}{d \omega} \ln \chi(\omega, 0)\right|_{\omega \rightarrow 0}$ manifested by Eq.(4) can be present even in the host SFL state (i.e., for $g=0$ ).

At low temperatures $(T \lesssim \Delta)$ the off-critical susceptibility may also differ between the "quantum disordered" (QD) and "renormalized classical" (RC) regimes [6]. In either case, however, it can be cast in the form of a sum over the intermediate $(1,2, \ldots$-fermion $)$ states, the coherent (one-particle) component $\chi_{c o h}^{a b}(\omega, q)$ assumes a form similar to $\chi_{Q C}^{a b}(\omega, q)$ where $\chi_{0}^{a b}(\Delta)$ is now given by the same expression as in Eq.(4) with the temperature substituted by $\Delta$, while the relaxation rate $\gamma_{Q D, R C} \propto T e^{-\Delta / T}$ $[6,7]$ is suppressed as compared to its QC counterpart.

In contrast to the pole-like structure of $\chi_{c o h}^{a b}(\omega, q)$, a generic incoherent two-particle contribution

$$
\chi_{i n c}^{a b}(\omega, q)=\frac{\eta^{a b}(q)}{\left(v^{2} q^{2}-\omega^{2}\right)^{\beta_{a b}}\left(v^{2} q^{2}+4 \Delta^{2}-\omega^{2}\right)^{1-\beta_{a b}}}
$$

features either a singularity $\left(\beta_{a b}<1\right)$ or a cusp $\left(\beta_{a b}>1\right)$ at the $\omega=2 \Delta$ threshold.

In what follows, we consider a representative example of the spin-isotropic system supporting massive spinons that is provided by the frustrated Heisenberg model $\left(J_{i j}^{a}=J \delta_{j, i \pm 1}+J^{\prime} \delta_{i, j \pm 2}, \quad B_{i}^{a}=0\right)$.

In this model (which can also be mapped onto a twochain zigzag ladder), a sufficiently strong $\left(J^{\prime}>J_{c}^{\prime} \approx\right.$ $0.24 J$ ) next-nearest-neighbor (NNN) exchange gives rise to a spontaneous dimerization and opens up a gap $\Delta \propto$ $J \exp \left(-\right.$ const. $\left.J / J^{\prime}-J_{c}^{\prime}\right)$.

For all $J^{\prime} \leq J_{c}^{\prime}$ the system remains in the same critical state as the standard NN Heisenberg model described by the $S U(2)_{k=1}$ Wess=Zumino-Witten theory. Accordingly, the susceptibility given by the hydrodynamical expression $\chi_{c o h}^{a b}(\omega, q)=\delta^{a b} v^{2} q^{2} /\left(v^{2} q^{2}-\omega^{2}\right)$ shows neither a gap, nor an anomalous exponent.

The effect of the NNN and other frustrating exchange interactions which render spinons massive can also be studied in the framework of the sine-Gordon (SG) model where the spinons can be identified with the $\mathrm{SG}$ (anti)solitons [8].

The incipient SFL instability with respect to dimerization occurs when the (anti)solitons become degenerate with some of their bound states ("breathers"), thus resulting in the formation of massive triplets (prototypes of "flying qutrits").

In the gapped regime, the low- $T$ susceptibility takes the form (5) where $\eta_{a b}(q) \propto q^{2}$ and $\beta_{a b}=\frac{3}{2} \delta_{a b}$, thereby indicating that the forward scattering remains non-singular and the (anti)solitons largely preserve their integrity. This expectation is supported by our calculation of the spinon decoherence rates (see Eq.(12) below).

Nonetheless, by analogy with the stability criteria of the conventional Fermi liquid, one might expect that a 
singular (long-ranged and/or retarded) spin fluctuationmediated interaction could make the spinons ill-defined and give rise to a critical "spinon non-Fermi liquid" state characterized by anomalous exponents.

The subsequent analysis shows that such a behavior can indeed occur in spin-anisotropic systems, a popular exactly solvable example of which is presented by the $X Y$-model in transverse field $\left(J_{i j}^{x}=-J \frac{1}{2}(1+\right.$ $\left.\delta) \delta_{j, i \pm 1}, J_{i j}^{y}=-J \frac{1}{2}(1-\delta) \delta_{j, i \pm 1}, B_{i}^{z}=B\right)$.

After the Jordan-Wigner transformation followed by the Bogoliubov rotation, the $X Y$-model with $\delta \neq 0$ gets transformed into a free gas of Majorana fermions with the velocity $v=J \delta$ and the gap $\Delta=B-J$ that vanishes at the Ising QCP [6].

These excitations possess no conserved physical spin and can be thought of as domain walls ( $Z_{2}$-kinks) between two locally degenerate ground states. The fact that the kinks lack a simple representation in terms of the constituent fermions $\psi_{k \sigma}$ implies that the corresponding one-spinon $\operatorname{RDM} \hat{\rho}(x-y, t)=<\psi_{\sigma}^{\dagger}(x, t) \psi_{\sigma^{\prime}}(y, t)>$ should exhibit strong decoherence (see Eq.(13) below).

In support of this expectation, the critical susceptibility of the model in question is now given by Eq.(3) where $\eta^{a b}(0) \neq 0$ and the anomalous exponents depend on $\delta$ (e.g., $\alpha_{x x}=3 / 4$ and $7 / 8$ for $\delta=0$ and 1 , respectively).

In the $\mathrm{QD}$ regime $(B>J)$, the functions $\chi^{x x, y y, x y}$ feature a pole-like structure, while in the $\mathrm{RC}$ domain $(B<J)$ they exhibit a kinematic square-root singularity $\left(\beta_{x x, y y, x y}=1 / 2\right)$ at the $2 \Delta$ threshold. In contrast, $\chi^{z z}$ displays a cusp $\left(\beta_{z z}=3 / 2\right)$ and remains incoherent for all values of the transverse field [9].

The unfying framework of the spin-fermion model (2) also allows one to investigate the effects of such qubits' imperfections as inhomogeneous local field or exchange couplings that vary from one site to another. In the presence of a static disorder, the $S U(2)$-invariant low-energy $\left(\omega, v q \lesssim \gamma_{t r}\right)$ susceptibility acquires a diffusive pole

$$
\chi^{a b}(\omega, q)=\frac{\chi_{u}^{a b} D_{s} q^{2}}{D_{s} q^{2}+i \omega}
$$

where the spin diffusion coefficient $D_{s}=v^{2} / 2 \gamma_{t r}$ is determined by the disorder-induced transport rate $\gamma_{t r}$. It is worth mentioning that in a generic (non-integrable) spin chain $D_{s}$ is finite at $T>0$ even in the absence of disorder, consistent with a non-vanishing spin (thermal) resistivity of such systems [10].

Similar to the critical spin fluctuations, the soft diffusive mode can prodive for a possible source of singular forward scattering between the spinons (see Eq.(14) below).

In either case, the effect of the low-energy spinfluctuation exchange on the spinon evolution operator $\hat{U}^{R}(x, t)$ can be studied in the framework of the continuum equation

$$
\left[i \partial_{t}-\epsilon\left(i \partial_{x}\right)+i \hat{\sigma}^{a} S^{a}(x, t)\right] \hat{U}^{R}(x, t \mid \vec{S})=\hat{\mathbf{1}} \delta(x) \delta(t)
$$

where $\vec{S}(x, t)$ represents an instantaneous configuration of the effective spin environment that the propagating spinon is subjected to.

The formal solution to Eq.(7) can be cast in the form

$$
\hat{U}(x, t \mid \vec{S})=\int_{0}^{\infty} d \tau \sum_{k} \int \frac{d E}{2 \pi} e^{i k x-i E t-\tau\left(E-\epsilon_{k}\right)}
$$

$P \exp \left[i \int_{0}^{\tau} e^{i \tau^{\prime}\left(\omega-\epsilon_{k+q}+\epsilon_{q}\right)} d \tau^{\prime} \sum_{q} \int \frac{d \omega}{2 \pi} \hat{\sigma}^{a} S^{a}(\omega, q)\left(e^{i q x}-1\right)\right]$

where the matrix ordering $P$ is to be performed according to the time order in which the $\hat{\vec{\sigma}}$-matrices appear in the series expansion of the exponential operator.

Using Eq.(8) one can construct a one-spinon RDM given by the usual double-time Keldysh path integral $\hat{\rho}(x-y, t \mid \vec{S})=\int d u d v \hat{U}^{R}(x-u, t \mid \vec{S}) \hat{\rho}^{(0)}(u-v) \hat{U}^{R}(v-$ $y,-t \mid \vec{S})$ for a given realization of the spin environment and an initial density matrix $\hat{\rho}^{(0)}(x)$.

Upon statistically averaging $\hat{\rho}(x-y, t \mid \vec{S})$ over $\vec{S}(x, t)$, we arrive at the expression

$$
\hat{\rho}(x, t)=\int d y \hat{L}(x-y, t) \hat{\rho}^{(0)}(y) e^{-\Phi(x-y, t)}
$$

where we singled out the (super)operator of unitary evolution (hereafter $z=x-y+i 0$ )

$$
\begin{array}{r}
\hat{L}(z, t)=\hat{\mathbf{1}} \sum_{k} e^{i k z} \cos ^{2} \epsilon_{k} t= \\
\Delta I m \sum_{ \pm} \sqrt{\frac{z \pm v t}{z \mp v t}} K_{1}\left(\Delta \sqrt{z^{2}-v^{2} t^{2}}\right)
\end{array}
$$

At $\Delta=0$ the kernel $\hat{L}(z, t)=\hat{\mathbf{1}} \sum_{ \pm} \delta(z \pm v t)$ describes a mere ballistic spreading of the entanglement present in the initial state (a "non-relativistic" counterpart of this behavior was studied in the case of the ferromagnetic Heisenberg model in Ref. [11]).

Apart from the propagation, entanglement undergoes an apparent decay characterized by the decoherence factor

$$
\begin{gathered}
\Phi(z, t)=\frac{1}{2} \sum_{q} \int \frac{d \omega}{2 \pi} \frac{1-\cos \left(\omega-\epsilon_{k_{*}}+\epsilon_{k_{*}+q}\right) t}{\left(\omega-\epsilon_{k_{*}}+\epsilon_{k_{*}+q}\right)^{2}} \\
\operatorname{Im}^{a a}(\omega, q)\left(\operatorname{coth} \frac{\omega}{2 T}-\tanh \frac{\omega-\epsilon_{k}}{2 T}\right)(1-\cos q z)
\end{gathered}
$$

where $k_{*} \approx 1 / z$, and the spinon Fermi distribution function eliminates contributions of the bosonic modes with energies $\omega \gtrsim T$ which, otherwise, would have resulted in a residual decoherence at $T=0$.

Notably, Eqs.(9-11) bear a certain resemblance to the results of the semiclassical calculation of Refs. [10] where 
the one-particle RDMs of the exactly solvable transverse field Ising model and $O(3)$ non-linear $\sigma$-model were shown to factorize into a product of a quantum propagator and a relaxation factor, the latter becoming trivial at $T=0$.

Now, estimating Eq.(11) on the semiclassical trajectory $x-y=v t$, we can deduce a characteristic decoherence rate $\Gamma$ from the equation $\Gamma=\left.\frac{d}{d t} \Phi(v t, t)\right|_{t=1 / \Gamma}$.

In the spin-isotropic case, we obtain

$$
\begin{array}{rr}
\Gamma_{\text {sym }} \propto g^{2} T, & T>\Delta \\
\propto g^{2} \frac{T^{2}}{\Delta} e^{-\Delta / T}, & T<\Delta
\end{array}
$$

which is comparable to or smaller than the SFL relaxation rates $\gamma_{Q C, Q D, R C}$ caused by a non-singular scattering neglected in Eq.(2), thus suggesting that the spinons remain well-defined elementary excitations.

By contrast, in the totally anisotropic case the decoherence rate is strongly enhanced as compared to Eq.(12)

$$
\begin{array}{rr}
\Gamma_{\text {asym }} \propto g^{2} J^{2 \alpha} T^{1-2 \alpha} & T>\Delta \\
\propto g^{2} \frac{J^{2 \alpha} T^{2}}{\Delta^{1+2 \alpha}} e^{-\Delta / T}, & T<\Delta
\end{array}
$$

The decoherence rates given by Eq.(13) become greater than the SFL ones at $\max [T, \Delta]<T_{*} \propto g^{1 / \alpha} J$ which signals a rapid decay of any spinon-like wave packet with a definite spin $S=1 / 2$, thus indicating the difficulty of creating robust flying qubits with the spin-anisotropic Hamiltonians.

At temperatures below $T_{*}$ the analysis of decoherence requires one to proceed beyond the lowest order of the cluster expansion employed in the derivation of Eqs.(911).

Besides, Eqs. $(12,13)$ demonstrate that the decoherence exhibited by the one-spinon RDM can be suppressed by the spectral gap and, conversely, it tends to become stronger as the system is tuned towards a QCP. This conclusion complements the earlier analysis of the decohering effect of an external dissipative environment which, unlike in the present case, remains unchanged [12].

The decoherence rate also appears to be enhanced in the disordered case where the susceptibility takes the form (6)

$$
\Gamma_{d i f} \propto g^{4 / 3} T^{2 / 3} \gamma_{t r}^{1 / 3}
$$

The diffusive contribution (14) dominates over its ballistic counterpart (12) at all temperatures up to $T_{* *} \propto$ $\gamma_{t r} / g^{2}$ where both terms are of order $\gamma_{t r}$, while below $T_{* * *} \propto g^{4} \gamma_{t r}$ the rate $\Gamma_{\text {dif }}$ becomes of order $T$ and a systematic account of higher orders of the cluster expansion is again required.

Besides being manifested by the single-spinon RDM, the rate (14) controls various amplitudes that are sensitive to the spinon phases such as, e.g., the "spinCooperon" amplitude associated with the pairs of timereversed trajectories that return to the starting point.
Thus, $\Gamma_{\text {dif }}$ can also be extracted from the weaklocalization correction to the low-temperature spin (thermal) conductivity

$$
\delta \sigma_{s} \sim \int_{\tau_{t r}}^{\infty}\left(\frac{D_{s}}{t}\right)^{1 / 2} e^{-\Phi(v t, t)} d t \propto\left(D_{s} \Gamma_{d i f}\right)^{1 / 2}
$$

that should be contrasted with the lowest order (Drude) result $\sigma_{s}^{(0)} \propto D_{s} \chi_{u}$.

It was argued in Refs. [13] that in the QIP context the qubits' localization by disorder may prove advantageous, as far as the goal of creating well-localized spin- $1 / 2$ states is concerned. However, our results show that increasing the amount of disorder would come at a price of greater decoherence rates.

To summarize, in the present work we studied the apparent spin decoherence that occurs at $T>0$ in even a closed non-integrable 1D spin system. This short-time "deterministic decoherence" can be revealed by, e.g., the RDMs of a finite number of spinons propagating in a dissipative environment created by the rest of the interacting qubits.

We focused on the near-critical and diffusive regimes where the emergence of soft collective modes (critical and diffusive, respectively) is more likely to cause a singular forward spinon scattering and an ensuing rapid decay of the spinon RDMs.

In the case of a spin-isotropic Eq.(1) and no disorder, we found that spinon remains as good of a coherent elementary excitation as it is in the parent SFL model. By contrast, in the spin-anisotropic case and/or in the presence of spin diffusion the decoherence rates are strongly enhanced, consistent with the fact that the system does not support coherent spin- $1 / 2$ states with definite momenta. We also observed that the decoherence rates can be reduced by tuning the system away from an incipient criticality and/or localization.

Lastly, our findings suggest that the enhanced decoherence (as quantified by the spinon RDMs) in the nearcritical and diffusive regimes might significantly reduce any possible benefits of both the emergent massive entanglement [4] and the disorder-induced localization of the flying qubits' [13] that have been recently discussed as potentially attractive features of the spin chain-based QIP designs.

This research was supported by ARO under Contract DAAD19-02-1-0049 and by NSF under Grant DMR0071362 .

[1] E. Barouch, B. M. McCoy, and M. Dresden, Phys. Rev. A2, 1075 (1970); E. Barouch and B. M. McCoy, 
ibid A3, 786 (1971); L. Amico and A. Osterloh, condmat/0306285.

[2] M. A. Nelson and I.L.Chuang, Quantum Computation and Quantum Information (Cambridge University Press, Cambridge, 2000).

[3] M. Hermele, M. P. A. Fisher, and L. Balents, condmat/0305401.

[4] A. Osterloh et al, Nature 416, 608 (2002); T. J. Osborne and M. A. Nielsen, Phys. Rev. A66, 032110 (2002); G. Vidal et al, Phys. Rev. Lett. 90, 227902 (2003).

[5] A. J. Millis, Phys. Rev. 45, 13047 (1992); Ph. Monthoux and D. Pines, ibid B47 6069 (1993).

[6] S. Sachdev, Quantum phase transitions (Cambridge University Press, Cambridge, 1999).

[7] T. Jolicoeur and O. Golinelli, Phys. Rev. B50, 9265 (1994).
[8] D. G. Shelton, A. A. Nersesyan, and A. M. Tsvelik, Phys. Rev. B53, 8521 (1996); D. Allen, F. H.L Essler, and A. A. Nersesyan, ibid B61, 8871 (2000).

[9] J.S. Caux, F.H.L. Essler, U. Loew, Phys. Rev. B68, 134431 (2003).

[10] S. Sachdev and K. Damle, Phys. Rev. Lett. 78, 943 (1997); S. Sachdev and A.P. Young, ibid 78, 2220 (1997);

K. Damle and S. Sachdev, Phys. Rev. B57, 8307 (1998);

C. Buragohain and S. Sachdev, ibid B59, 9285 (1999).

[11] J. S. Pratt and J. H. Eberly, Phys. Rev. B64, 195314 (2001).

[12] D. V. Khveshchenko, cond-mat/0301111, to appear in Phys. Rev. B.

[13] L. F. Santos and M. I. Dykman, quant-ph/0303130; condmat/0308026; L. F. Santos, G. Rigolin, and C. O. Escobar, quant-ph/0310177. 